\title{
Guest Editorial: Special Issue on ACCV 2018
}

\author{
C. V. Jawahar ${ }^{1} \cdot$ Hongdong $\mathrm{Li}^{2} \cdot$ Greg Mori ${ }^{3} \cdot$ Konrad Schindler ${ }^{4}$
}

Published online: 25 February 2020

(c) Springer Science+Business Media, LLC, part of Springer Nature 2020

This special issue consists of papers ranging from both classical multi-view geometry to recent deep learning methods deep stereo matching networks, GAN and adversarial learning, and multi-task learning for video object segmentation.

The idea for a special issue about architectures and theories for computer vision came from the ACCV conference held in 2018. There were 979 submissions to ACCV, of which 269 were accepted and presented at the conference in Perth. A selection of papers ranked highest in the review process by the over 1000 reviewers and 34 Area Chairs were then reviewed again by a Best Paper Prize committee to determine the winners and runners-up for AFCV's three awards, the Saburo Tsuji Best Paper Award, the Sang Uk Lee Best Student Paper Award and the Songde Ma Best Application Paper Award.
We, the program chairs of ACCV 2018 and guest editors of this special issue, invited the authors of the ACCV'18 award-winning papers to submit extended manuscripts to this special issue. These extended papers underwent a second rigorous peer-review process before they are accepted to this special issue.

As guest editors we would like to thank all authors for their valuable contributions to this special section. We also thank the reviewers, who made helpful suggestions that further improved the quality of the papers, and the editorial staff of IJCV for their support. We hope that the papers in this special sec-tion will become a useful resource for researchers and practitioners in computer vision.

Publisher's Note Springer Nature remains neutral with regard to jurisdictional claims in published maps and institutional affiliations.
Hongdong $\mathrm{Li}$

hongdong.li@anu.edu.au

C. V. Jawahar

jawahar@iiit.ac.in

Greg Mori

mori@cs.sfu.ca

Konrad Schindler

schindler@ethz.ch

1 IIIT - Hyderabad, Hyderabad, India

2 Australian National University, Canberra, Australia

3 Simon Fraser University, Vancouver, Canada

4 ETH Zürich, Zurich, Switzerland 